\title{
The Transition from Slow to Fast Solar Wind: Charge State Composition and Electron Observations
}

\author{
S. Hefti ${ }^{1}$, T. H. Zurbuchen ${ }^{1}$, L. A. Fisk ${ }^{1}$, G. Gloeckler ${ }^{1}$, \\ D. Larson ${ }^{2}$, and R. P. $\operatorname{Lin}^{2}$ \\ ${ }^{I}$ Department of Atmospheric, Oceanic and Space Sciences, University of Michigan, Ann Arbor, USA \\ ${ }^{2}$ Space Sciences Laboratory, University of California, Berkeley, USA
}

\begin{abstract}
The charge state composition is a very sensitive measure for the electron properties at around 2 solar radii heliocentric distance. Even though the basic principle of the charge-state freeze-in process is rather well understood, it is not clear how the non-thermal properties of the electron distribution functions influence the frozen-in charge states. The obvious question to ask, then, is whether in situ electrons exhibit the same temporal variations as the charge state composition. Using SWICS/ACE and 3DP/WIND data, we find that the core electrons are dominated by local effects like compression and have no memory of their coronal origin. Supra-thermal electrons, on the other hand, show a clear correlation with the charge state data.
\end{abstract}

\section{INTRODUCTION}

Charge state distributions of solar wind minor ions are a measure of the thermal properties of the electrons around a few solar radii, where direct electron temperature measurements are difficult. Using ionization and recombination rates known from laboratory measurements (e.g. (1)), the temperature at the freeze-in location can be inferred (6). It is an ongoing discussion whether the charge-state inferred temperature is an appropriate measure of the coronal temperature. Measurements of emission lines of coronal ions with SUMER/SOHO indicate significantly lower temperatures than are expected from charge-state data (10); (4).

In situ measurements of electrons provide information about the local plasma properties and the thermal properties at the low corona (9). At low energies, the electron distribution functions are dominated by dynamic processes like heating by compression. At higher energies, the collision frequencies drop dramatically, and supra-thermal electrons propagate essentially scatter-free from the low corona to $1 \mathrm{AU}$. These electrons should therefore carry the coronal imprint.

In this paper we present a detailed comparison of in situ electrons and high resolution charge state measurements. It is, to our knowledge, the first comparison of electron and charge state data. We use composition data from SWICS/ACE (3) and electron data from 3DP/WIND (7) during early 1998 when WIND and ACE were both close to L1.

\section{DATA REDUCTION}

SWICS is a mass- and charge spectrometer optimized to measure the elemental and ionic composition of the solar wind. It employs electrostatic energy per charge filtering, time-of-flight measurement, and a solid state detector to determine the residual energy of solar wind ions. From these measurements, the speed, thermal speed and density of each ion can be deduced. The SWICS instrument is described in more detail in (3).

The reduction of SWICS/ACE data relies on a forward model which accurately calculates the time-of-flight (TOF) and solid state detector energy ( $E_{S S D}$ ) for given solar wind properties and instrument settings (5). Densities are then obtained by essentially counting the events falling into a region in TOF $-E_{S S D}$ space. A typical count rate for $\mathrm{O}^{7+}$, the lower abundant of the oxygen ions, is $\approx 20$ events per instrument cycle, resulting in a typical statistical uncertainty for the highest time resolution data of the $\mathrm{O}^{7+} / \mathrm{O}^{6+}$ ratio of $20 \%$.

For a detailed description of the 3DP/WIND instrument and data reduction, see (7).

\section{DATA}

Slow and fast solar wind exhibit distinctly different charge state compositions. In order to see a clearly defined signal, we have selected two periods where slow is followed by fast solar wind. In both cases, the transition occurs on a time scale of hours. The change of solar wind 


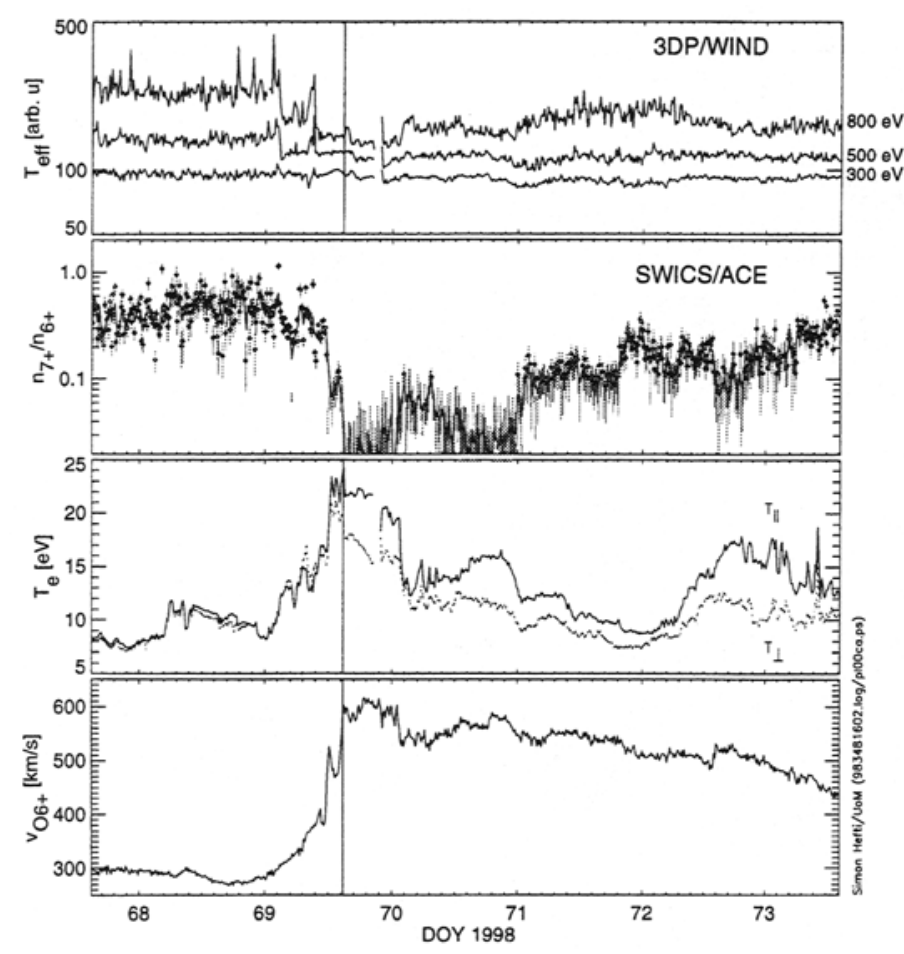

FIGURE 1. Example 1 (8 - 14 Mar 1998). Plotted are the solar wind speed $v$, the average temperature of the electrons $T_{e}$ measured by 3DP/WIND, the ratio of $\mathrm{O}^{7+}$ and $\mathrm{O}^{6+}$ measured by SWICS/ACE, and the effective temperature $\mathrm{T}_{\text {eff }}$ of the suprathermal electrons. The correlation between $T_{\text {eff }}$ and $\mathrm{O}^{7+} / \mathrm{O}^{6+}$ is surprisingly good.

speed is accompanied by a change of the ratio of $\mathrm{O}^{7+}$ and $\mathrm{O}^{6+}$ which is a sensitive measure for coronal conditions, and is also accompanied by a change of the iron to oxygen ratio. This is typical for the transition from slow wind originating from equatorial regions with predominantly closed magnetic field lines to fast, coronalhole type solar wind (e.g. (2)). The two figures present the speed of $\mathrm{O}^{6+}$, the average electron temperature $T_{e}$, the ratio $\mathrm{O}^{7+} / \mathrm{O}^{6+}$, and a measure for the temperature of the supra-thermal electrons, $\mathrm{T}_{\text {eff }}$, discussed below. In the case of $\mathrm{O}^{7+} / \mathrm{O}^{6+}$ we plot the data points obtained from the best time resolution of the instrument of $12 \mathrm{~min}$, together with the statistical uncertainty. Overplotted as a solid line is the running mean with a window of $24 \mathrm{~min}$.

Notice first the well-known anti-correlation of the solar wind speed $v$ and the $\mathrm{O}^{7+} / \mathrm{O}^{6+}$ ratio shown in Figure 1 . Fast solar wind usually emerges from relatively cool coronal holes with an open magnetic field configuration. Slow solar wind, on the other hand, usually originates from hotter regions with predominantly closed magnetic field lines. Notice then the apparent anticorrelation of the $\mathrm{O}^{7+} / \mathrm{O}^{6+}$ ratio and the average electron temperature $T_{e}$. Clearly, the core electrons have no memory of the coronal temperature and are dominated by local effects like heating in the compression region. It is also noteworthy that the electron distribution changes across the compression region, as is indicated by the separation of $\mathrm{T}_{\|}$(solid line) and $\mathrm{T}_{\perp}$ (dotted line).

The effective temperature of the supra-thermal electrons at a particular energy can be derived from their distribution function $f$ with

$$
T_{\text {eff }}=\frac{-1}{k[d \ln f / d E]}
$$

where $E$ is the electron energy and $k$ Boltzmann's constant. In the case of a Maxwellian distribution function $f=\exp \left[-\frac{E}{k T}\right]$, eq 1 yields $T_{\text {eff }}=T$, the temperature. The top panel of Figure 1 shows the effective temperature determined from electrons propagating along the magnetic field away from the Sun at 300,500 , and $800 \mathrm{eV}$. The supra-thermal electron data exhibits a time series which is surprisingly similar to the $\mathrm{O}^{7+} / \mathrm{O}^{6+}$ data.

Figure 2 shows the same data for a different period. Again, the slow solar wind is followed by a high-speed stream which is clearly identified by both the $\mathrm{O}^{7+} / \mathrm{O}^{6+}$ as well as the $\mathrm{Fe} / \mathrm{O}$ ratio. As in Figure 1, we find an apparent anti-correlation between the $\mathrm{O}^{7+} / \mathrm{O}^{6+}$ ratio and the average temperature of the electrons, $T_{e}$. The correlation between $\mathrm{O}^{7+} / \mathrm{O}^{6+}$ and $\mathrm{T}_{\text {eff }}$, on the other hand, is remarkably good. In this case, almost every detail of the charge state data is also seen in the supra-thermal electrons.

\section{DISCUSSION}

We compare high resolution charge state data from SWICS/ACE and three-dimensional electron distribution functions from 3DP/WIND. We use two time periods of transitions between slow and fast wind during mid-March and end of May in 1998, when WIND and ACE were close to L1.

The transitions from slow to fast solar wind have very clear signatures in both the $\mathrm{O}^{7+}$ to $\mathrm{O}^{6+}$ and the $\mathrm{Fe} / \mathrm{O}$ ratio, as previously discussed by (2). $\mathrm{O}^{7+} / \mathrm{O}^{6+}$ is quite variable in time. In the slow solar wind, it typically fluctuates around 0.3 . In the fast solar wind, it is normally below 0.1 , and clear structures can be distinguished which are related either to changing solar wind expansion or different source regions of the solar wind.

The transition from slow to fast wind occurs on a time- 


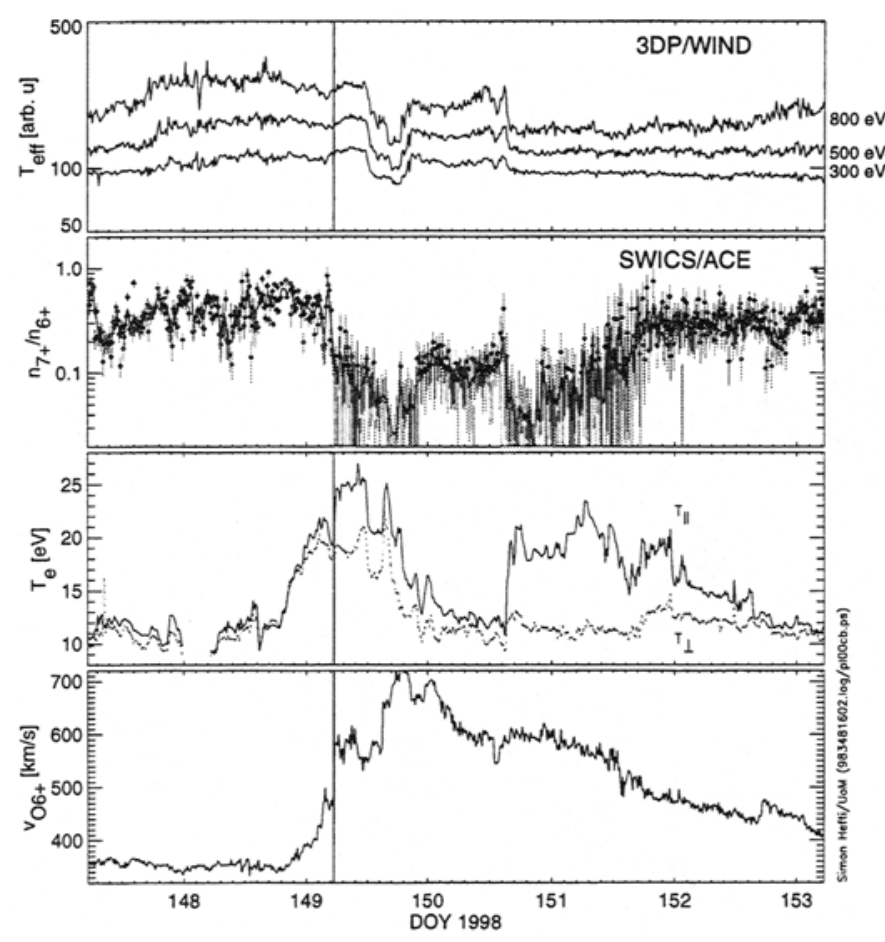

FIGURE 2. Example 2 (27 May - 2 Jun 1998). As Figure 1. tribution functions should show a clear correlation to the freeze-in data. This is in fact the case. Often, even small features in the electrons are remarkably well recovered. Notice that the propagation time of supra-thermal electrons and minor ions may differ by several days. Where there is very good correlation, the field-lines had to be stationary on that time-frame. On the other hand, periods of phase-shifts are likely to imply time-dependence of the field-lines between the freeze-in and the electron acceleration.

This result demonstrates a close association of suprathermal electrons with freeze-in data.

\section{REFERENCES}

1. Arnaud and Rothenflug, $A A S, 60425-457$ (1985).

2. Geiss et al., Science, 268, 1033-1036 (1995).

3. Gloeckler et al., Space Sci. Rev., 86, 495, (1998).

4. Hefti, S., PhD Thesis, University of Bern, 1997.

5. Hefti, S., Technical Report, University of Michigan, 1998.

6. Hundhausen, A.J., Coronal Expansion and Solar Wind, Springer (1972).

7. Lin et al., Solar Phys., 100, 537 (1995).

8. Marsch et al., in Physics of the Inner Heliosphere, Springer (1990).

9. Scudder, J.D., and Olberts, S., JGR, 84, 2755 (1972).

10. Wilhelm et al., Solar. Phys.,170, 75-104 (1997). scale of less than a few hours, from fast to slow wind on a time-scale of the order four days.

Since the electron distribution function in the corona is the main factor determining the frozen-in charge states of the solar wind ions, we are interested in the in situ electron data. We find no correlation between the average electron temperature $T_{e}$ and the charge state data, however. Because $T_{e}$ is dominated by the core electrons with a typical energy of $10 \mathrm{eV}$ at $1 \mathrm{AU}$, we conclude that the core electrons measured in situ are strongly affected by the dynamic processes in the solar wind, consistent with (9). This can be seen particularly in the compression region at the slow-fast interface (Figures 1 and 2). The actual value of the average electron temperature $T_{e}$ seems to be similar in both solar wind regimes. Only the anisotropy $T_{\|} / T_{\perp}$ shows a clear dependence on the solar wind type.

Supra-thermal electrons, on the other hand, propagate essentially scatter-free from the low corona to $1 \mathrm{AU}$ (8). Accordingly, the effective temperature $\mathrm{T}_{\text {eff }}$ calculated from the supra-thermal portion of the electron dis- 